Research Paper: Neuroscience

\title{
Specific amplifications and copy number decreases during human neural stem cells differentiation towards astrocytes, neurons and oligodendrocytes
}

\author{
Ulrike Fischer ${ }^{1}$, Ella Kim², Andreas Keller ${ }^{3}$ and Eckart Meese ${ }^{1}$ \\ ${ }^{1}$ Department of Human Genetics, Saarland University, Homburg/Saar, Germany \\ 2 Translational Neurooncology Research Group, Johannes Gutenberg University, Mainz, Germany \\ ${ }^{3}$ Clinical Bioinformatics, Saarland University, Saarbrücken, Germany \\ Correspondence to: Ulrike Fischer, email: ulrike.fischer@uks.eu \\ Keywords: gene amplification, CDK4, MDM2, EGFR, astrocytes, Neuroscience \\ Received: September 14,2016 Accepted: February 27, $2017 \quad$ Published: March 07, 2017
}

Copyright: Fischer et al. This is an open-access article distributed under the terms of the Creative Commons Attribution License (CC-BY), which permits unrestricted use, distribution, and reproduction in any medium, provided the original author and source are credited.

\section{ABSTRACT}

There is growing evidence that gene amplifications are an attribute of normal cells during development and differentiation. During neural progenitor cell differentiation half of the genome is involved in amplification process. To answer the question how specific amplifications occur at different stages and in different lineages of differentiation we analyzed the genes CDK4, MDM2, EGFR, GINS2, GFAP, TP53, DDB1 and MDM4 in human neural stem cells that were induced to differentiate towards astrocytes, neurons and oligodendrocytes. We found specific amplification pattern for each of the eight analyzed genes both in undifferentiated neural stem and progenitor cells and in cells that were induced for differentiation. Different amplification patterns were also found between adherently grown neural stem cells and cells that were grown as spheres. The most frequently amplified genes were MDM2 and CDK4 with the latter amplified in all three lineages at all analyzed stages. Amplification of the analyzed genes was also found in four glioma stem-like cells. The combined amplification data of stem cells and of tumor stem cells can help to define cell populations at the origin of the tumor. Furthermore, we detected a decrease of gene copies at specific differentiation stages most frequently for MDM4. This study shows specific amplification pattern in defined stem cell populations within specific time windows during differentiation processes indicating that amplifications occur in an orderly sequence during the differentiation of human neural stem and progenitor cells.

\section{INTRODUCTION}

There is ample evidence for gene amplifications in human tumors $[1,2]$. More recently, several studies reported gene amplification also in stem cells or progenitor cells during developmental processes and specifically during differentiation. As part of the embryonic development $E R B B 2$ gene amplification occurs in human trophoblast cells [3]. Recently, amplification of placental genes was reported in trophoblast giant cells [4]. We found a larger number of amplifications using array-CGH and fluorescence in situ hybridization during differentiation of human neural progenitor cells and mouse neural stem and progenitor cells $[5,6]$. We also detected gene amplifications during the differentiation of human and mouse myoblasts towards muscle cells [7]. Amplifications during the differentiation process occur apparently only in small sub-population of the cells [5] making them difficult to detect especially in high throughput assays, which mostly analyze a large number of cells. Although the presence of amplifications as part of developmental process appears to be assured, the biological role of amplifications in this physiological process is less well established. As for many mutations, amplifications can be a driving force or a bystander for these processes. With only a few cells carrying amplifications, it is near to impossible to obtain evidence for functional relevance by determining the expression levels of the amplified 
genes within a cell population that mostly contains cells without gene amplification. Alternatively, amplifications that occur in an orchestrated way during specific cellular processes may be indicative of functional relevance as opposed to amplifications that occur randomly. Our abovementioned studies on the differentiation of human and mouse myoblasts towards muscle cells provided first evidence for ordered amplification events. Here, we set out to answer the question whether amplifications occur in an orderly sequence as part of the differentiation of human neural stem cells. To this end, we compared the sequence of amplification events during three different lineages of differentiation and ask for the specificity of an amplification pattern for each of these processes. In detail, we differentiated neural stem cells towards astrocytes, neurons and oligodendrocytes to investigate gene amplifications.

\section{RESULTS}

An overview on experimental design is shown in Figure 1. To analyze amplifications during different lineages of differentiation we induced differentiation of adherent growing human neural stem cells (NSC; H9 hESC-derived; GIBCO) into oligodendrocytes, astrocytes, and neurons. In detail, NSC were grown as adherent cells on CELL Start ${ }^{\mathrm{TM}}$ treated culture surface with EGF and $\mathrm{bFGF}$ for $24 \mathrm{~h}$ in the following referred to as time point 0 h. Subsequently, NSC cells were induced to differentiate towards oligodendrocytes with Neurobasal $\AA$ medium supplemented with B-27® Serum-Free Supplement, GlutaMAX ${ }^{\mathrm{TM}}-\mathrm{I}$ and $\mathrm{T} 3$ on polyornithine and laminincoated culture dish. Differentiation towards neurons was induced by Neurobasal ${ }^{\circledR}$ medium supplemented with B-27® Serum-Free Supplement and GlutaMAX ${ }^{\text {TM}}-\mathrm{I}$ on polyornithine- and laminin-coated culture dish. Differentiation towards astrocytes was induced by D-MEM supplemented with N-2, GlutaMAX ${ }^{\mathrm{TM}}-\mathrm{I}$, and $1 \%$ FBS on Geltrex ${ }^{\circledR}$ matrix-coated culture dish. Spontaneous differentiation was induced by growth factor depletion. In each of the four assays DNA was isolated four times after 24 hours each (1-4 days). For all lineages of differentiation and all time points we determined the copy number of eight genes including $C D K 4, M D M 2$, EGFR, GINS2, GFAP, TP53, DDB1 and MDM4 all of which are known to localize to amplified genomic regions in neural progenitor cells during differentiation and to be amplified in human glioblastoma. The amplification was determined by qPCR analysis (TaqMan) in four replicates with the data analyzed by the software "copy caller" (Applied Biosystems) as described previously [7, 8]. Mean calculated copy numbers for control DNA from blood lymphocytes revealed values in the range from 1.8 to 2.14 and were further regarded as normal diploid copy number. A decreased copy number was defined by values $<1.8$, an increased copy number by values $>2.2$ and $<2.3$ and an

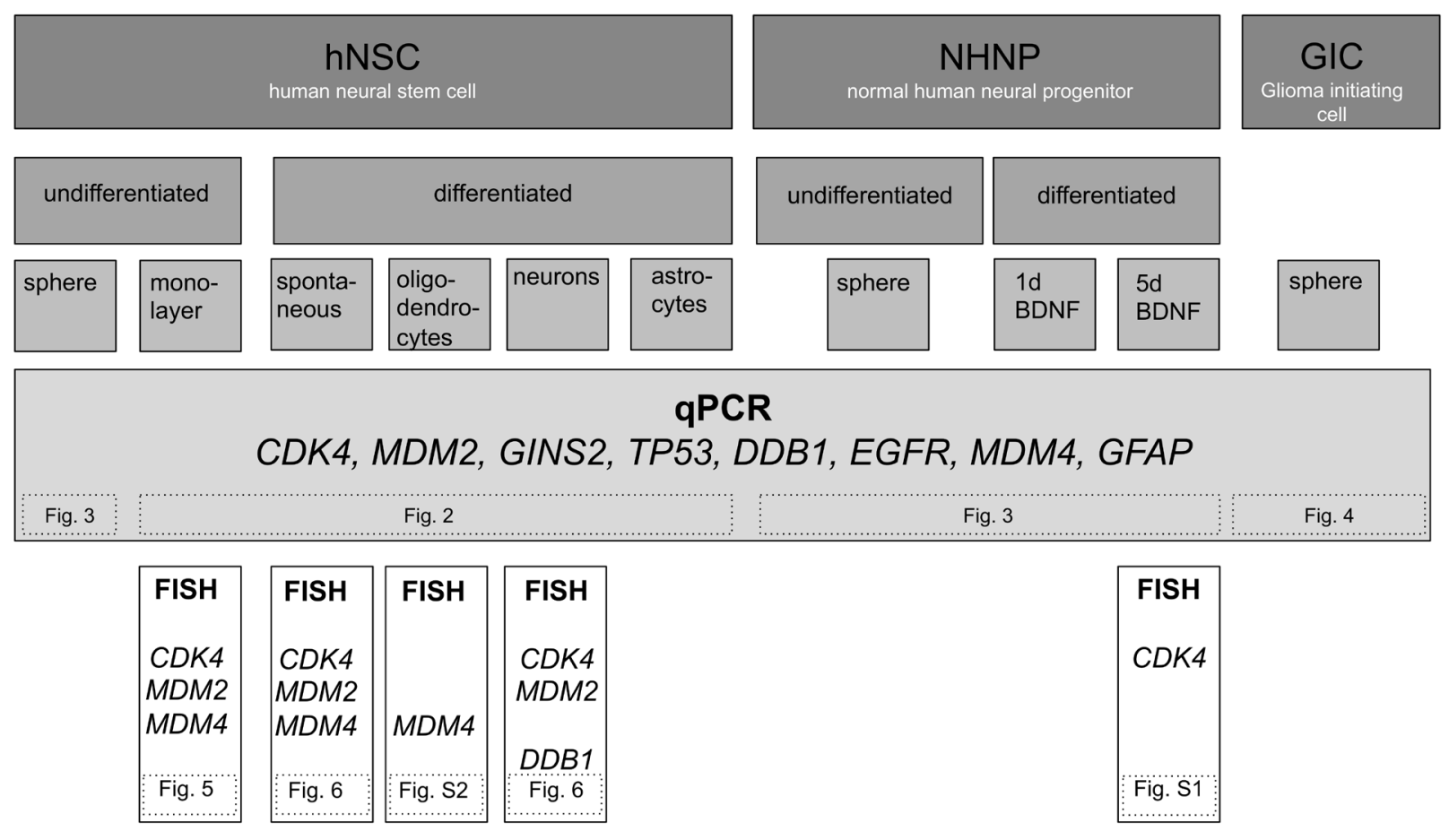

Figure 1: Overview of experimental design. Graphic overview on used cells, differentiation induction, and techniques to analyze amplification. Reference to other figures, is given by numbers in dashed boxes. 
Table 1: Results of copy number analysis in neural stem and progenitor cells

\begin{tabular}{|c|c|c|c|c|c|c|c|c|}
\hline & CDK4 & MDM2 & GINS2 & TP53 & DDB1 & EGFR & MDM4 & GFAP \\
\hline PB & 2 & 2 & 2 & 2 & 2 & 2 & 2 & 2 \\
\hline $\mathrm{NSC} 0 \mathrm{~h}$ & 2,36 & 2,13 & 2,1 & 2,09 & 1,52 & 1,88 & 2,36 & 2,34 \\
\hline spontan $24 \mathrm{~h}$ & 3,28 & 2,4 & 2,49 & 2,31 & 2,18 & 2,09 & 1,88 & 2,08 \\
\hline spontan $48 \mathrm{~h}$ & 3,13 & 2,6 & 2,26 & 2,27 & 2,19 & 2,03 & 1,94 & 2,03 \\
\hline spontan $72 \mathrm{~h}$ & 2,71 & 2,55 & 2,25 & 2,27 & 2,18 & 2,1 & 1,86 & 2,03 \\
\hline spontan $96 \mathrm{~h}$ & 2,86 & 2,42 & 2,25 & 2,23 & 2,28 & 1,97 & 1,95 & 2,02 \\
\hline Oligo $24 \mathrm{~h}$ & 3,35 & 2,1 & 2,33 & 2,01 & 1,83 & 1,6 & 1,99 & 2,06 \\
\hline Oligo $48 \mathrm{~h}$ & 2,9 & 2,26 & 1,94 & 1,93 & 1,75 & 1,59 & 1,55 & 1,76 \\
\hline Oligo $72 \mathrm{~h}$ & 2,6 & 2,15 & 1,96 & 2,02 & 1,92 & 1,77 & 1,57 & 1,74 \\
\hline Oligo $96 \mathrm{~h}$ & 3,35 & 2,59 & 2,21 & 2,14 & 2,07 & 2,02 & 1,76 & 1,92 \\
\hline Neu $24 \mathrm{~h}$ & 3,19 & 2,48 & 2,33 & 2,27 & 2,25 & 1,82 & 1,95 & 2,02 \\
\hline Neu $48 \mathrm{~h}$ & 3,2 & 2,67 & 2,12 & 2,17 & 2,26 & 1,89 & 1,75 & 1,93 \\
\hline Neu $72 \mathrm{~h}$ & 2,56 & 2,51 & 2,1 & 2,08 & 2,02 & 1,91 & 1,62 & 1,82 \\
\hline Neu 96h & 2,53 & 2,5 & 2,11 & 2,17 & 2,16 & 2,02 & 1,68 & 1,89 \\
\hline Astro $24 \mathrm{~h}$ & 3,11 & 2,73 & 2,25 & 2,29 & 2,3 & 2,16 & 1,74 & 1,96 \\
\hline Astro $48 \mathrm{~h}$ & 3,15 & 2,49 & 2,26 & 2,25 & 2,33 & 2,07 & 1,75 & 1,92 \\
\hline Astro $72 \mathrm{~h}$ & 2,61 & 2,76 & 2,18 & 1,88 & 2,22 & 2,07 & 1,68 & 1,92 \\
\hline Astro $96 \mathrm{~h}$ & 2,58 & 2,46 & 2,26 & 2,21 & 2,22 & 2,02 & 1,91 & 2,02 \\
\hline NSC sphere & 3,21 & 2,06 & 2,58 & 2,23 & 2,15 & 1,94 & 2,36 & 2,22 \\
\hline NHNP sphere & 3,1 & 2,33 & 2,49 & 2,45 & 2,62 & 2,53 & 2,08 & 2,32 \\
\hline BDNF 1d & 2,63 & 2,18 & 2,33 & 2,26 & 2,48 & 2,22 & 1,85 & 1,98 \\
\hline BDNF 5d & 2,94 & 2,29 & 2,41 & 2,35 & 2,59 & 2,5 & 1,98 & 2,08 \\
\hline
\end{tabular}

Mean calculated copy numbers from four replicate experiments were defined as follows: copy number increase: 2.2-2.3 (light green), amplification: $>2.3$ (dark green), under- replication: $<1.8$ (orange)

amplification by values $>2.3$. Results of all experiments were summarized in Table 1.

As for CDK4 the copy number was already amplified in undifferentiated NSCs $(0 \mathrm{~h})$ as compared to peripheral blood that was used as reference. We found a high copy number increase of CDK4 $24 \mathrm{~h}$ after differentiation induction in all 4 differentiation protocols. In all protocols the CDK4 copy number decreased at $72 \mathrm{~h}$. For the last time point ( $96 \mathrm{~h}$ ) we found divergent results with a strong increase of the copy number in differentiating oligodendrocytes, a moderate increase in the spontaneously differentiated cells, and a low copy number in differentiating astrocytes and neurons (Figure 2a and Table 1). MDM2 that is localized in close vicinity to $C D K 4$ on chromosome 12 , showed a different amplification pattern upon differentiation induction. Among the 4 differentiation protocols the less MDM2 amplification was found in NSCs differentiating towards oligodendrocytes. Differentiating oligodendrocytes showed the strongest $M D M 2$ amplification after $96 \mathrm{~h}$. We found comparable $M D M 2$ amplification pattern in spontaneously differentiated cells and in differentiating neurons with the highest $M D M 2$ amplification levels found at $48 \mathrm{~h}$ and/or $72 \mathrm{~h}$. Differentiation into the astrocytic lineage showed yet a different pattern with the highest
MDM2 amplification levels at $24 \mathrm{~h}$ and $72 \mathrm{~h}$ (Figure $2 \mathrm{~b}$ and Table 1).

Similar to the amplification pattern of $C D K 4$, we found amplification for GINS2 already at $24 \mathrm{~h}$ specifically in differentiating oligodendrocytes, differentiating neurons and spontaneously differentiated cells. Subsequently there was a lower GINS2 copy number at $48 \mathrm{~h}$ and at $72 \mathrm{~h}$. At the last time point $(96 \mathrm{~h})$ there was again an increased copy number of GINS2 in differentiating oligodendrocytes while the copy number remained unchanged in spontaneously differentiated cells and differentiating neurons (Table 1). A yet different amplification pattern was found for TP53. While spontaneously differentiated cells showed a similarly increased copy number at all 4 time points, differentiating oligodendrocytes showed no copy number increase. Both differentiating neurons and differentiating astrocytes showed the highest copy numbers at the beginning $(24 \mathrm{~h})$ of the observed time period (Table 1). Like for the abovementioned genes including CDK4, MDM2, GINS2 and TP53 we found specific pattern of copy number changes for $D D B 1, E G F R$, $M D M 4$ and GFAP for each of the four differentiating protocols. However, the genes DDB1, EGFR, MDM4 and GFAP showed not only increased but also decreased copy numbers. As for the increased copy numbers, the 
a CDK4

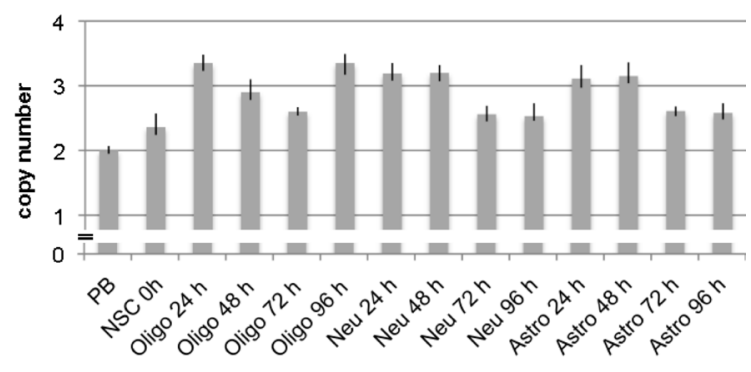

b MDM2

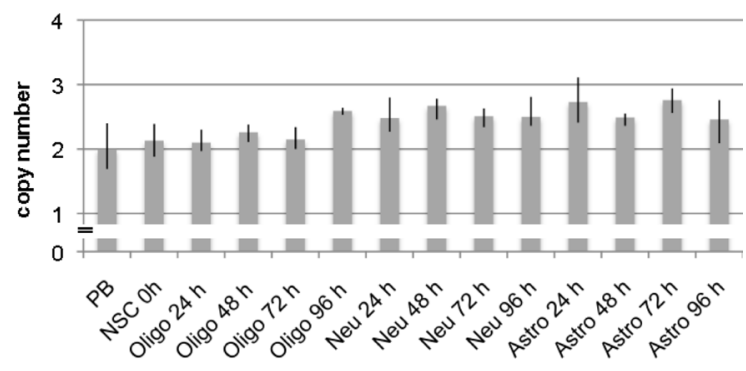

C DDB1

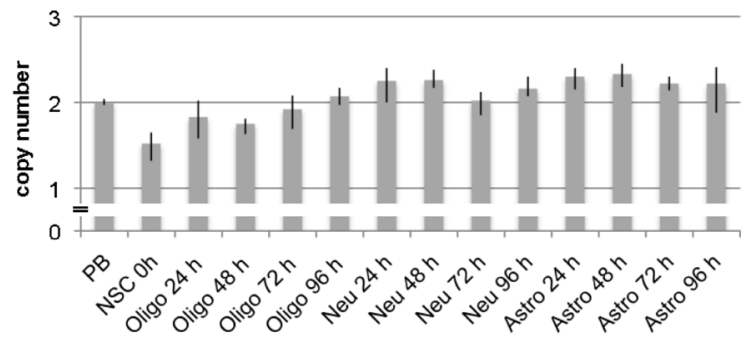

d MDM4

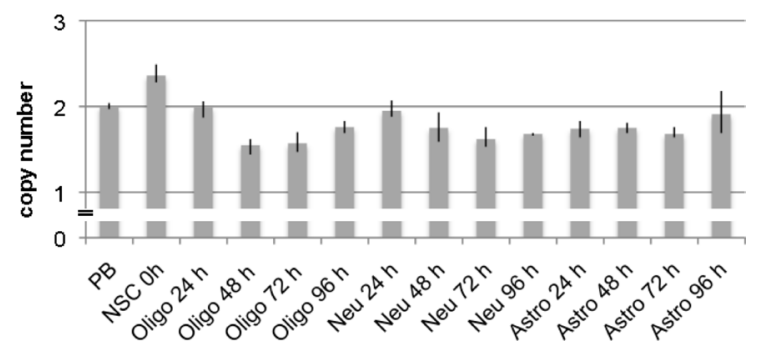

Figure 2: Copy number analysis of CDK4, MDM2, DDB1 and MDM4 using qPCR in neural stem cells and during differentiation. Copy number of $C D K 4, M D M 2, D D B 1$ and $M D M 4$ was analyzed by qPCR using TaqMan copy number assays. RNaseP was used as reference gene in the TaqMan assays and DNA from normal blood lymphocytes (PB) served as standard for normal diploid copy number. Copy numbers are shown as mean from four technical replicates with vertical lines indicating the range. NSC cells (oh) served as undifferentiated control. Differentiation induced NSC cells were analyzed at four time points after differentiation induction. Differentiation induction was either towards oligodendrocytes (oligo), towards neurons (neu) and towards astrocytes (astro). CDK4 was amplified with the highest copy number after $24 \mathrm{~h}$ and $96 \mathrm{~h}$ of differentiation towards oligodendrocytes and after $24 \mathrm{~h}$ and $48 \mathrm{~h}$ differentiation towards neurons and astrocytes a. $M D M 2$ was amplified with the highest copy number after differentiation towards neurons and astrocytes $\mathbf{b} . D D B 1$ revealed a decreased copy number in undifferentiated NSCs and after $48 \mathrm{~h}$ differentiation towards oligodendrocytes whereas an increased copy number was detected after differentiation towards neurons and astrocytes $\mathbf{c}$. MDM4 was amplified only in undifferentiated NSCs whereas a decreased copy number was detected from $48 \mathrm{~h}$ differentiation towards oligodendrocytes and neurons and from $24 \mathrm{~h}$ differentiation towards astrocytes d. 
a

CDK4

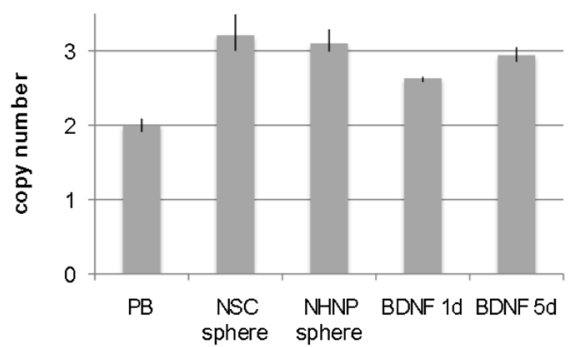

C

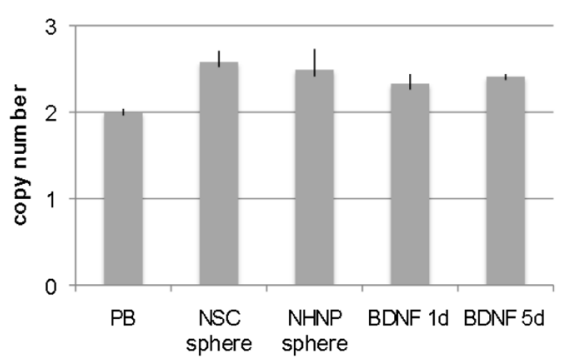

e

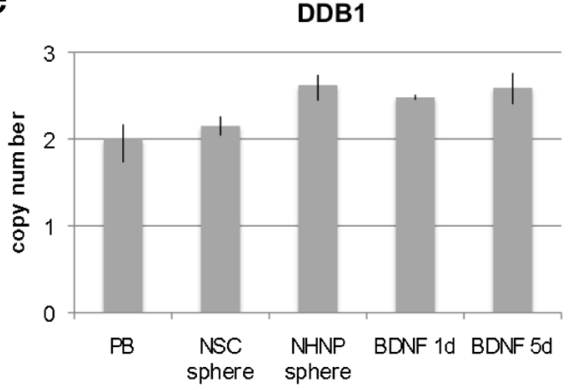

g

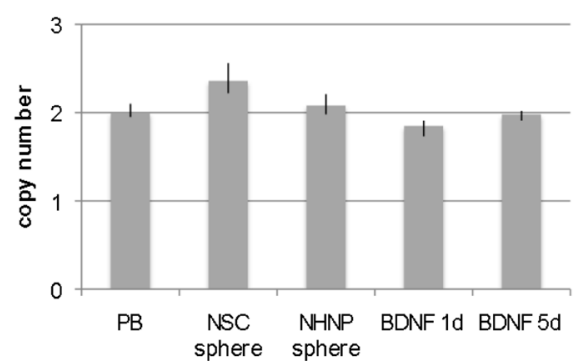

b

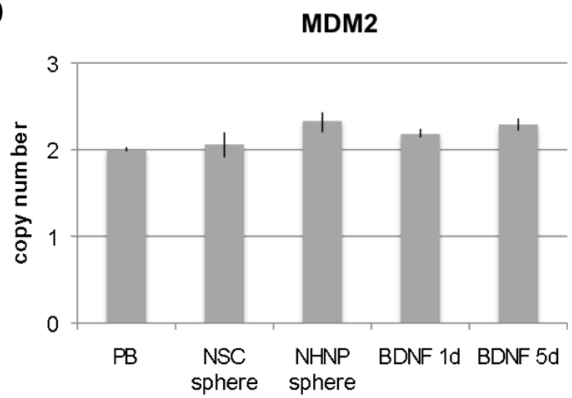

d

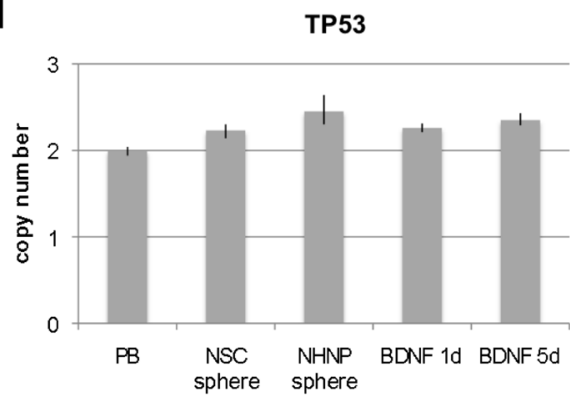

f

EGFR

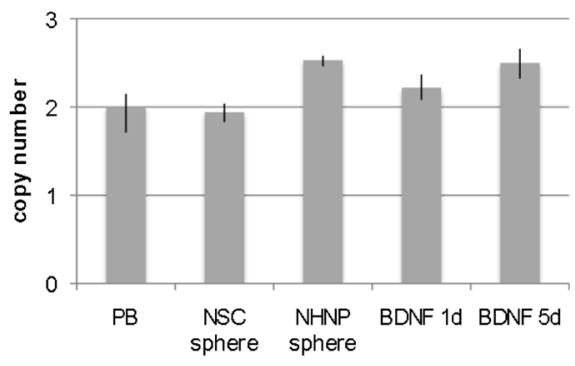

h

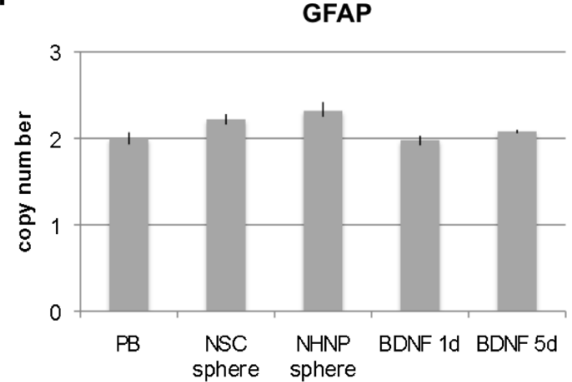

Figure 3: Copy number analysis in neural stem cell spheres and progenitor cell spheres. Copy number of $C D K 4, M D M 2$, GINS2, TP53 DDB1, EGFR, MDM4 and GFAP was analyzed by qPCR using TaqMan copy number assays. RNaseP was used as reference gene in the TaqMan assays and DNA from normal blood lymphocytes (PB) served as standard for normal diploid copy number. Copy numbers are shown as mean from four technical replicates with vertical lines indicating the range. Differentiation induction of NHNP sphere cells was either for one day with BDNF (BDNF 1d) or five days with BDNF (BDNF 5d). NSC sphere cells and NHNP sphere cells serve as undifferentiated controls. CDK4 was amplified with equal copy number in NSC and NHNP sphere cells and after 5 days of differentiation of NHNP sphere cells with BDNF a. MDM2 was amplified in NHNP sphere cells and NHNP sphere cells differentiated for 5 days with BDNF but not in NSC sphere cells b. GINS2 was amplified with equal copy number in NSC sphere cells and NHNP sphere cells and in BDNF differentiated NHNP sphere cells c. TP53 was amplified in NHNP sphere cells and in 5-day differentiation induced NHNP cells but not in NSC sphere cells d. $D D B 1$ was amplified in NHNP sphere cells and differentiation induced NHNP cells but not in NSC sphere cells e. EGFR was amplified in NHNP sphere cells and in differentiation induced NHNP cells but not in NSC sphere cells f. MDM4 was amplified in NSC sphere cells but not in NHNP sphere cells and differentiation induced NHNP cells g. GFAP revealed an increased copy number in NSC sphere cells and amplification in NHNP sphere cells but not in differentiation induced NHNP cells $\mathbf{h}$. 
time points at which the decrease occurred were different between the affected genes. The copy number of $D D B 1$ was most decreased in undifferentiated NSCs (Figure 2c). We also found a decrease of the $D D B 1$ copy number in differentiating oligodendrocytes, specifically at $24 \mathrm{~h}, 48 \mathrm{~h}$, and $72 \mathrm{~h}$ (Table 1). As for EGFR, the most decreased copy number was found in differentiating oligodendrocytes, but a decrease in copy numbers of EGFR also occurred in differentiating neurons. Likewise, we found decreased copy numbers of GFAP in both differentiating oligodendrocytes and differentiating neurons. The overall strongest decrease in copy number was found for MDM4 with a decrease observed for all 4 differentiation-protocols (Table 1). While we found a decrease in copy number in differentiating oligodendrocytes, neurons and astrocytes, undifferentiated NSCs revealed MDM4 amplification (Figure 2d).

In addition to adherent growing cells growing as monolayer, cells growing as spheres were also analyzed. In detail we analyzed NSC and human neural progenitor cells (NHNP) (Lonza) both as undifferentiated spheres and NHNP spheres after differentiation induction with BDNF as described previously [5]. Both undifferentiated NSCs and NHNP spheres showed a similar pattern of $C D K 4$ copy number amplification (Figure 3a). The copy number in both sphere cultures was also higher than in undifferentiated NSCs that were grown as adherent cells. Upon differentiation the copy number of CDK4 in NHNP spheres was decreased especially after $24 \mathrm{~h}$ as compared to the undifferentiated NHNP spheres (Figure 3a). As for $M D M 2$ we did not detect an increase of the copy number in the NSC spheres. In contrast, we detected MDM2 amplification in NHNP spheres both in undifferentiated and in BDNF differentiation-induced NHNP spheres as shown in Figure 3b. For GINS2 we found amplifications in all spheres including NSC spheres, undifferentiated NHNP spheres, and BDNF differentiation-induced NHNP spheres (Figure 3c). Likewise a TP53 copy number increase occurred in all spheres with the strongest increase in undifferentiated NHNP spheres (Figure 3d). We also found specific pattern of copy number changes for $D D B 1$, EGFR, MDM4 and GFAP in NSC spheres, undifferentiated NHNP spheres, and BDNF differentiation-induced NHNP spheres. A comparable strong copy number decrease as observed for differentiating NSCs did not occur in cells that were grown as spheres. A minimal decrease was found only for $E G F R$ in NSC spheres and for $M D M 4$ in BDNF induced NHNPs (Figure 3f; 3g). By contrast, $D D B 1$ and $E G F R$ show a rather prominent amplification specifically in undifferentiated and in differentiationinduced NHNP spheres (Figure 3e; 3f). GFAP revealed a copy number increase in NSC spheres and amplification in undifferentiated NHNP sphere (Figure 3h).

\section{Amplifications in glioma stem-like cells}

In addition to neural stem cells and neural progenitor cells and their differentiation towards astrocytes, neurons and oligodendrocytes, we analyzed four glioma stem-like cells (GICs \#10, \#993, \#1043, G112) for amplification. While none of the GICs showed amplification for all genes, all samples showed amplifications for at least 3 genes. In detail, we found CDK4 amplification in samples GIC\#10, GIC \#993 and G112 as shown in Figure 4a. MDM2 amplification was found in GIC\#993 and GIC\#1043 but not in other lines as shown in Figure 4b. GINS2 amplification was detected in GIC\#10 and G112 as shown in Figure 4c. TP53 amplification was detected in GIC\#993 and G112 (Figure 4d). Interestingly, G112 harbors a mutant TP53 (mut p53, 273H) as described previously [9-11]. DDB1 was only amplified in GIC\#993 (Figure 4e). EGFR amplification was detected in all samples with the highest copy number in GIC\#10 (Figure 4f). MDM4 amplification was present in three samples (GIC\#10, GIC\#993 and G112) as shown in Figure 4g and GFAP amplification was detected in GIC\#993 and GIC \#1043 (Figure 4h).

\section{Confirmation of amplification/under- representation by FISH}

We used fluorescence in situ hybridization (FISH) as single cell based technique for confirmation of amplification and under-representation. Human neural stem cells were analyzed undifferentiated, spontaneously differentiated for $24 \mathrm{~h}$ and differentiation-induced towards neurons for 48 h. MDM4 amplification, that was detected by qPCR was confirmed by FISH in undifferentiated human neural stem cells. The MDM4 amplification was found with a very high number of fluorescence signals in $5 \%$ of neural stem cells. As control we used the centromere probe D1Z5 that maps to chromosome 1 like MDM4. The number of fluorescence signals of D1Z5 indicated a normal diploid copy number of this chromosome (Figure 5 $\mathrm{a}$ and $\mathrm{b}$ ). In addition, we confirmed $C D K 4$ amplification in $10 \%$ of undifferentiated neural stem cells. In $5 \%$ of $C D K 4-$ amplified cells we detected co-amplification of MDM2 as indicated by very prominent fluorescence signals (Figure $5 \mathrm{c}$ and $\mathrm{d}$ ).

FISH also confirmed $C D K 4$ amplification and MDM2 amplification in $15 \%$ of human neural stem cells that were induced to differentiate towards neurons for $48 \mathrm{~h}$ (Figure 6a). Furthermore, FISH confirmed DDB1 amplification in $10 \%$ of human neural stem cells that were induced to differentiate towards neurons for 48 h. Since $D D B 1$ maps to chromosome 11 we used $S T X 3$ that also maps to chromosome 11 as control. FISH with $S T X 3$ indicated a normal diploid copy number (Figure 6b). $C D K 4$ and $M D M 2$ co-amplification was detected 
a

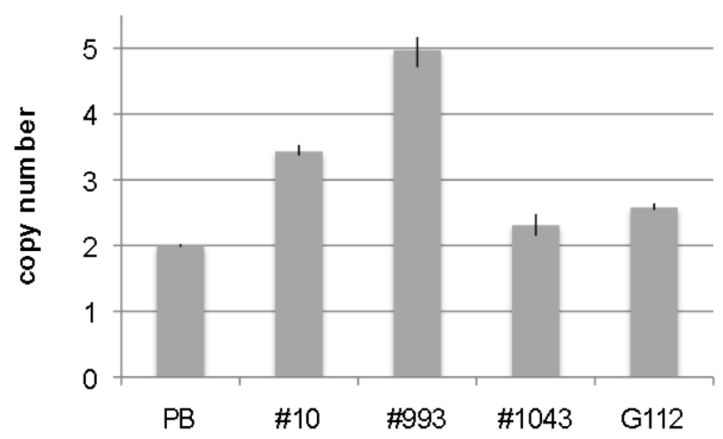

C

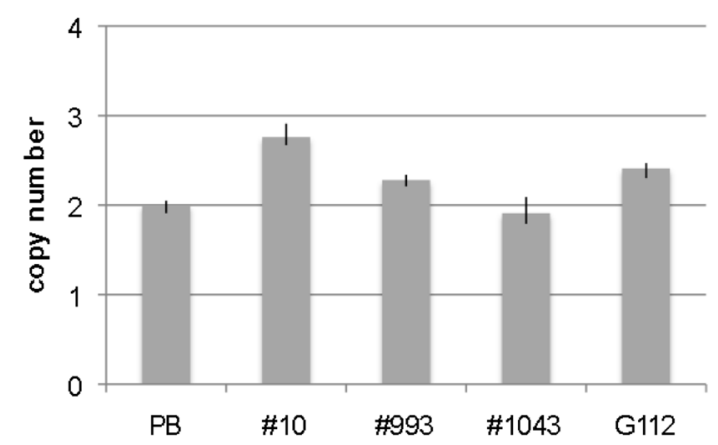

e

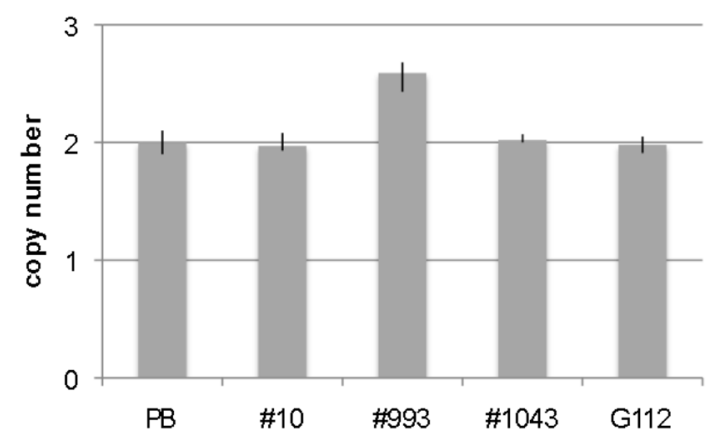

g

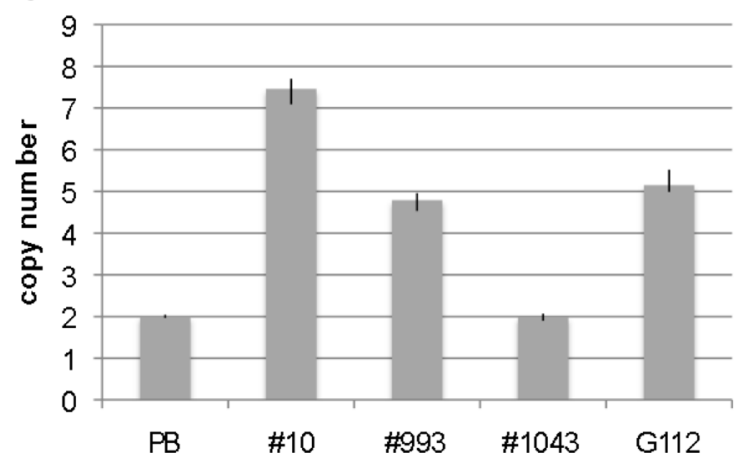

b

MDM2

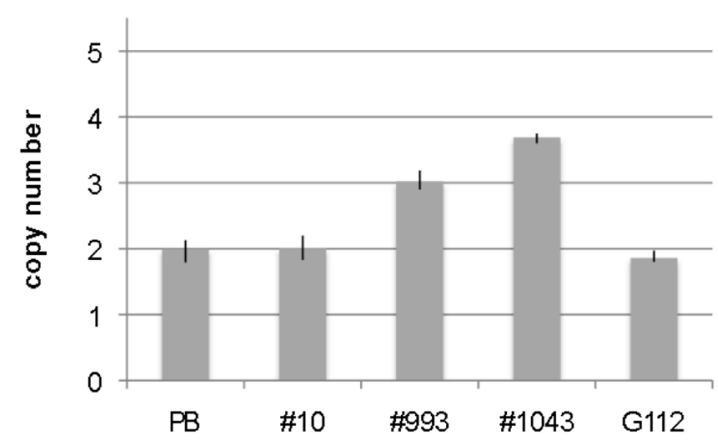

d

TP53

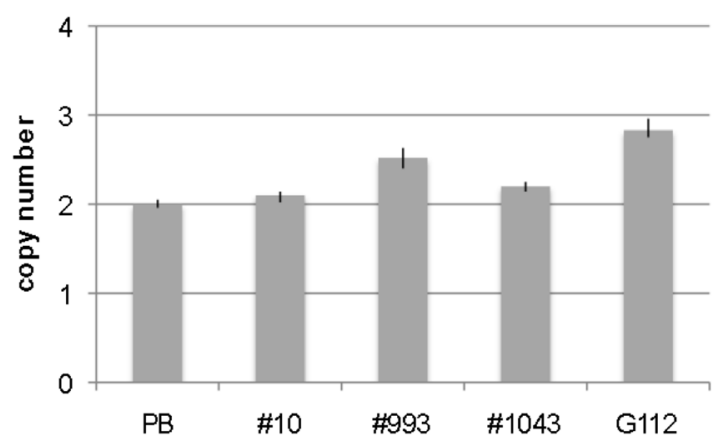

f

EGFR
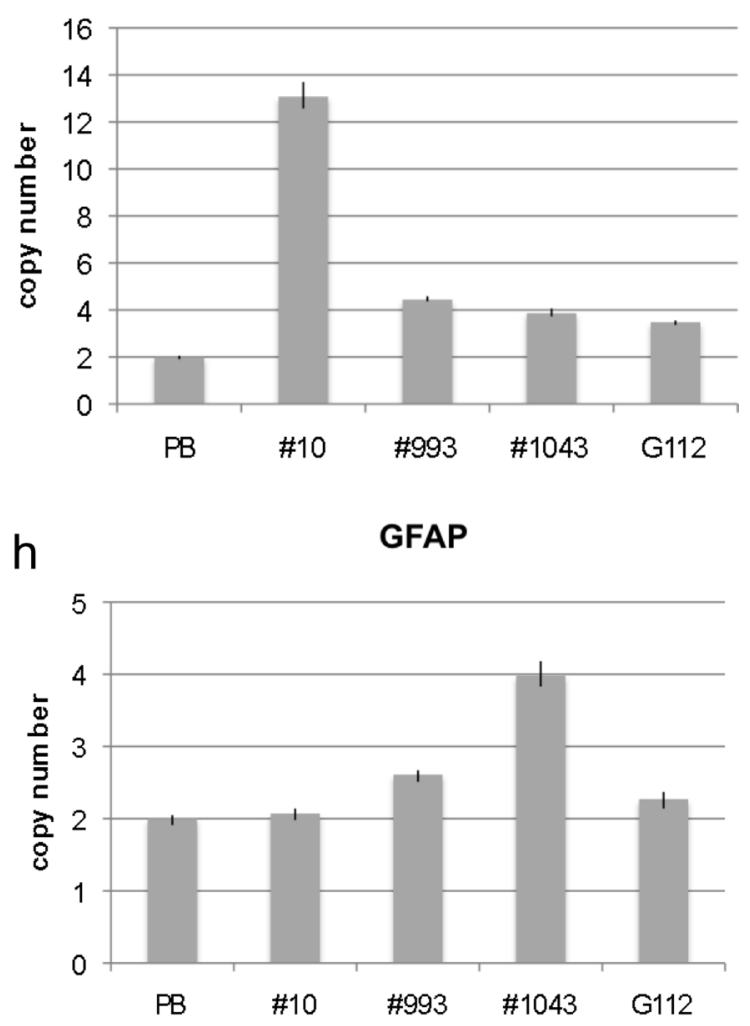

Figure 4: Copy number analysis of eight genes in glioma stem-like cells. Copy number of CDK4 a., MDM2 b., GINS2 c., TP53 d., $D D B 1$ e., $E G F R$ f., $M D M 4$ g. and GFAP h. was analyzed by qPCR using TaqMan copy number assays. Normal blood lymphocytes (PB) served as standard for normal diploid copy number. Copy numbers are shown as mean from four technical replicates with vertical lines indicating the range. 
in $1 \%$ of spontaneously differentiated neural stem cells, by FISH. In addition $25-30 \%$ of these cells also revealed $C D K 4$ amplification but a normal diploid copy number for $M D M 2$, which is localized in close vicinity of $C D K 4$ on chromosome 12 (Figure 6c).

As for spontaneously differentiated neural stem cells FISH identified MDM4 amplification in 2-5\% of the cells and under-representation in $20 \%$ of the cells with the latter cases indicated by a single MDM4 fluorescence signal per cell. FISH with the chromosome 1 centromere probe D1Z5 that was used as reference probe showed two signals indicating a normal diploid status (Figure 6d). FISH also revealed $C D K 4$ amplification in 5-day BDNF differentiation-induced NHNP cells and MDM4 under-representation in neural stem cells $48 \mathrm{~h}$ induced to differentiate towards oligodendrocytes (Supplementary Figures 1 and 2).

\section{DISCUSSION}

\section{Amplification and under-replication in context of stem cells}

Even undifferentiated neural stem cells harbor multiple gene amplifications as for example $M D M 4$ that was amplified in neural stem cells but showed a
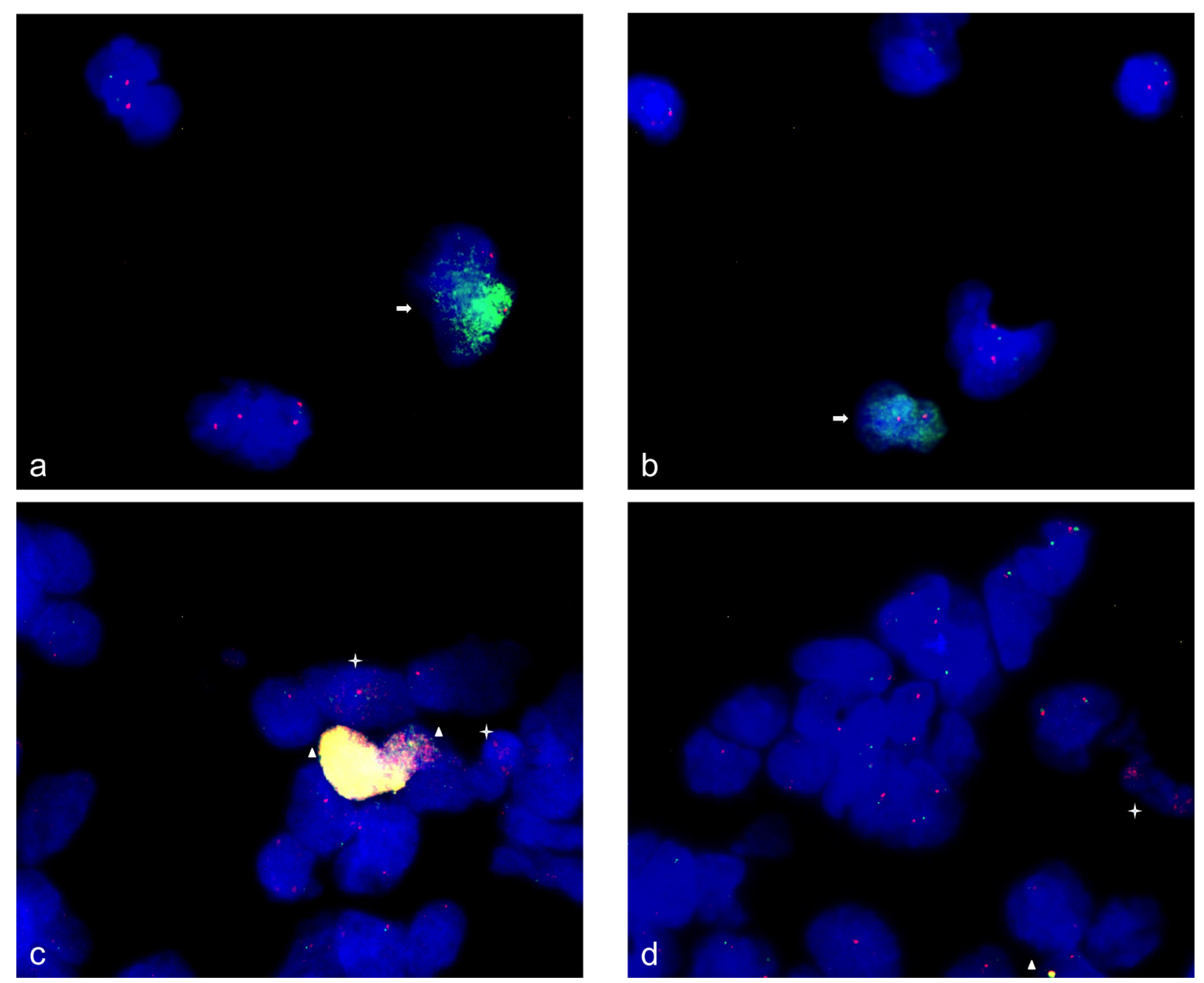

Figure 5: Gene amplifications on chromosomes 12q14-15 and 1q42.13 in human neural stem cells. FISH was used to analyze MDM4 (RP11-433N15) and CDK4 (RP11-571M6), MDM2 (RP11-611O2) gene amplification in human neural stem cells. 95\% of the neural stem cells revealed two signals for the chromosome alpha-centromere probe (D1Z5) on chromosome 1 (red) and MDM4 (green) whereas $5 \%$ of the neural stem cells revealed two signals for chromosome 1 alpha-centromere and high copy number of hybridization signals for MDM4 revealing an amplification of MDM4 a., b. Similarly $90 \%$ of neural stem cells revealed two signals for $C D K 4$ (red) and MDM2 (green) with approximately similar fluorescence intensity. In contrast $5 \%$ of neural stem cells revealed intense yellow hybridization signals indicating co-amplification of $C D K 4$ and $M D M 2$ and $5 \%$ of neural stem cells revealed enhanced number of hybridization signals for $C D K 4$ and two signals for $M D M 2$ indicating $C D K 4$ amplification c., d. Representative cells with amplifications are marked by arrow (MDM4), asterisk (CDK4) and triangle (CDK4 and $M D M 2)$. Nuclei were counterstained with DAPI. 
clearly decreased copy number in neural stem cells that were differentiated towards oligodendrocytes, neurons and astrocytes. Similar copy number decreases have been described in the context of gene amplifications in Drosophila [12] and most recently in mouse trophoblast cells [4]. Other genes like $C D K 4$ were amplified not only in undifferentiated neural stem cells and undifferentiated neural progenitor cells but also during the induced differentiation processes of these cells. Notably, the amplification process appears not due to an altered polyploidy since both genes CDK4 and MDM2 are mapping on chromosome 12. Chromosome 12 shows a normal copy number in neural stem cell spheres. The copy number changes that are found for chromosome 12 during differentiation are not related to the copy number changes found for $C D K 4$. Similar results that confirm amplification independent from polyploidy were found for TP53 and GFAP both localized on chromosome 17, $D D B 1$ and $S T X 3$ (control probe in FISH experiments) both localized on chromosome 11 and for MDM4 that revealed either amplification and/or under-replication accompanied with diploid copy number of chromosome 1.

Different amplification patterns were also found between adherently grown neural stem cells and cells that were grown as spheres. A difference of the amplification level was for example found between the genes GINS2 and $D D B 1 . D D B 1$ was under-replicated in adherent neural stem cells but had normal copy number in spheres. GINS2
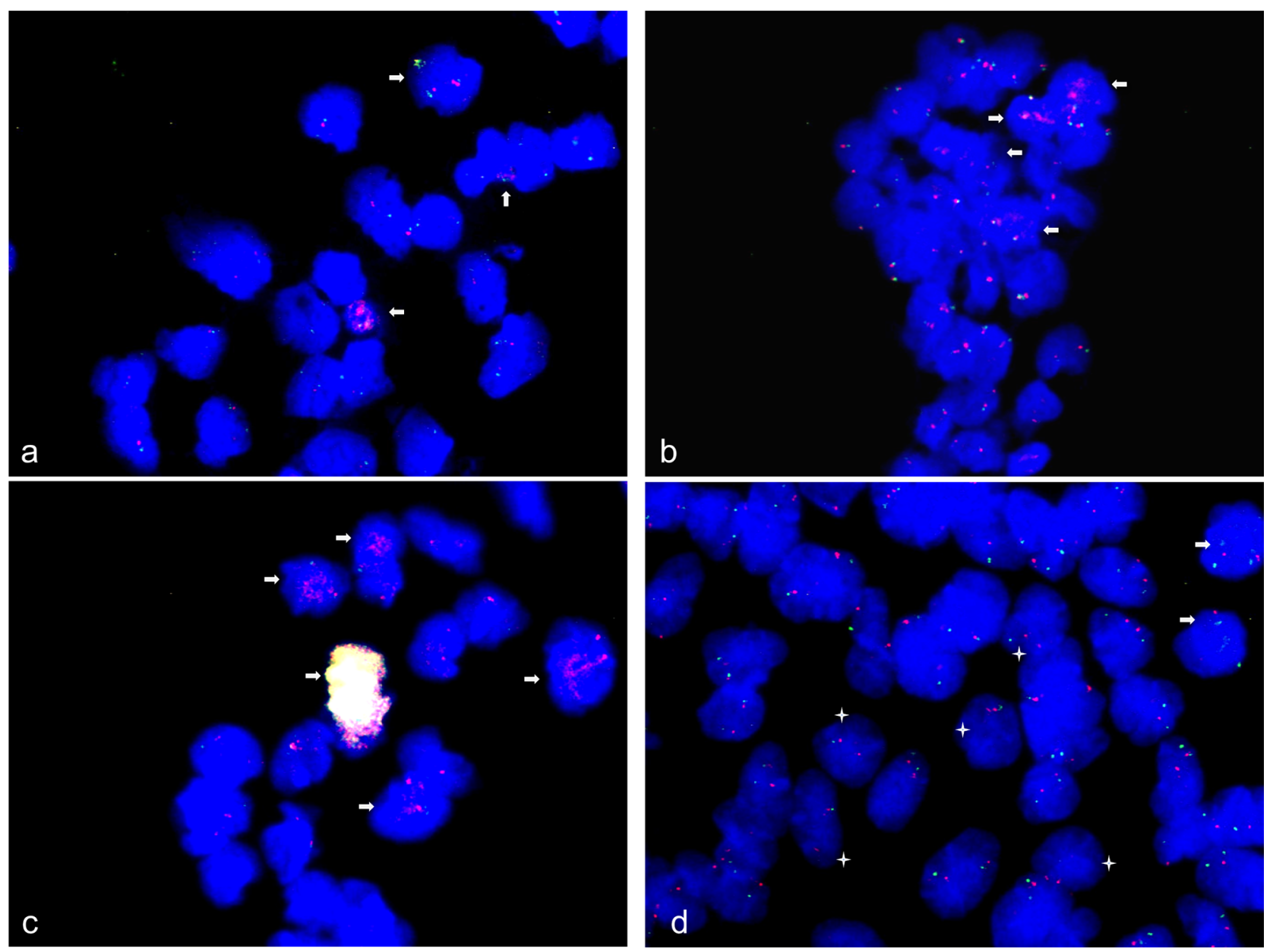

Figure 6: Gene amplification and under-replication in spontaneous and neural differentiation induced human neural stem cells. FISH was used to analyze CDK4 (RP11-571M6), MDM2 (RP11-611O2), DDB1 (CTC-820M2) and MDM4 (RP11-433N15) copy number in human neural stem cells induced to differentiate spontaneous for $24 \mathrm{~h}$ and towards neurons for $48 \mathrm{~h}$. CDK4 (red) and MDM2 (green) amplifications were detected in $15 \%$ of neural stem cells induced to differentiate towards neurons for $48 \mathrm{~h}$ a. $D D B 1$ amplification (red) was detected in $10 \%$ neural stem cells induced to differentiate towards neurons for $48 \mathrm{~h} \mathrm{~b}$. and remaining cells revealed two copies for $D D B 1$ and $S T X 3$ control BAC probe (green; RP11-468F15). CDK4 and MDM2 high copy co-amplification (yellow/white fluorescence signals) was detected in $1 \%$ of cells spontaneously differentiated and in additional $25-30 \%$ of cells $C D K 4$ amplification was detected accompanied with normal MDM2 copy number (green) c. Under-replication of MDM4 was detected in $20 \%$ of cells differentiated spontaneously for $24 \mathrm{~h}$ with only one fluorescence signal (green) accompanied with normal copy number of alpha-centromere probe chromosome 1 (red) and amplification of MDM4 was detected in less than 5\% of those cells d. Representative cells with amplifications are marked by arrow and cells with under-replication are marked by asterisk. Nuclei were counterstained with DAPI. 
showed a normal copy number in adherent neural stem cells but was amplified in spheres. Differences in the amplification between spheres formed by neural stem cells or neural progenitor cells were also found for $E G F R$, TP53, MDM2 and DDB1.

As for the biological function of the amplified genes, both GINS2 and DDB1 are involved in replication. While GINS2 belongs to the GINS complex that has an essential role in the initiation of DNA replication and for the progression of DNA replication forks [13], $D D B 1$ is involved in replication control through ubiquitination and degradation of CDT1 [14]. TP53 and DDB1 are involved in DNA damage response. MDM2 and MDM4 are p53regulating proteins with complementary but distinct roles in the regulation of TP53 activities. Both contain a p53-binding domain and are thought to be involved in suppression of TP53 transactivation and apoptotic function. Since the TP53 amplification was predominantly detected in neural progenitor cells and in neural stem cells differentiating towards neurons and astrocytes or towards neural and glial cells, TP53 amplification is likely to be an attribute of differentiating cells. This observation is consistent with the observation by Meletis and colleagues reporting that TP53 suppresses self-renewal in adult neural stem cells [15]. Furthermore, TP53 up-regulation contributes to maintaining genome integrity in progenitor cells during differentiation steps that are prone to genome instability. Interestingly, we found a very high amplification level for the GFAP gene in neural stem cells and neural progenitor cells despite their lack of GFAP immunoreactivity for common anti-GFAP antibodies. Vice versa, no GFAP amplification was detected in differentiating neural stem or progenitor cells, which are characterized by increased expression of GFAP at the onset of differentiation. One possible explanation is that GFAP amplification may be associated with alternative splicing resulting in the expression of GFAP isoforms that may not be recognized by anti-GFAP antibodies commonly used in histological analyses. For example, one of the 8 known GFAP isoforms, GFAP 2 is highly expressed in radial glia and subventricular zone progenitors [16] but cannot be detected by commonly used anti-GFAP antibodies binding to the C-terminus which is different between GFAP $\partial$ and the most abundant isoform GFAP $\alpha$.

\section{Confirmation of amplification and/or under- replication}

There are limitations to all approaches tailored to detect copy number alterations including amplifications, polyploidy, deletions and under-replication. Both arrayCGH and NGS allow to obtaining an overview on copy number changes. Standard second-generation sequencing is unlikely to unravel the amplification patterns when only a fraction of the analyzed cells carry amplifications.
As for the array analysis, a threshold below $\log _{2} 0.8$ was frequently used to define deletions and a threshold higher $\log _{2} 1.2$ to define amplifications. While these thresholds were valuable for uniform cell populations they are of limited use for heterogeneous cell populations especially for analyzing small sub-populations carrying copy number alteration. For example our previous whole genome tiling array-CGH analysis detected $C D K 4$ amplifications in 5-days BDNF differentiated NHNP cells but not in 1-day BDNF differentiated NHNP cells [5]. Here, we choose threshold settings for qPCR analysis to identify copy number alterations that are limited to small sub-populations in heterogeneous cell populations. Comparable thresholds of $>2.31$ and $>2.5$ were used in studies on TERC gene amplification in chronic myeloid leukemia and EGFR copy number in metastatic lung cancer [17, 18]. In addition, we used fluorescence in situ hybridization to detect copy number alterations that occur in very few cells. For example, MDM4 underrepresentation in $24 \mathrm{~h}$ spontaneously differentiated hNSC cells was not detected by qPCR analysis even using our threshold settings but by FISH, which revealed loss of one copy in $20-25 \%$ of the cells.

Notably, we did not detect copy number decreases by qPCR in sphere cells. This may be due to the fact that sphere cells were only gradually exposed to differentiation inducing conditions. Depending on sphere size the relative number of cells with copy number changes is lower than in cell culture. While gene amplifications with a high copy number increase to more than 50 copies are still detectable in spheres, copy number decreases with only one copy lost per cell, go very likely undetected in spheres. The potential of alternative whole genome sequencing to detect copy number changes is controversially discussed [19]. Tattini and co-workers indicated third generation sequencing as potential solution, which in our opinion may be ideally combined with single cell sequencing.

\section{Amplification in context of glioma cells}

$E G F R$ is amplified in almost $50 \%$ of glioblastoma and $20 \%$ of anaplastic oligodendroglioma. Previously, we did not detected EGFR amplifications in undifferentiated NHNP sphere cells by whole genome tiling array-CGH analysis only showing $\log _{2}$-ratio values that did not reach the threshold for amplification [5]. Likewise, array-CGH did not detect EGFR amplifications in human and mouse neural progenitor cells neither in a differentiated nor in a not-differentiated state. By contrast, other genes including CDK4 and MDM4 frequently amplified in glioblastoma were also amplified in human neural progenitor cells during differentiation [5]. Using TaqMan PCR analysis we now show EGFR amplifications in human neural progenitor cells and in human neural progenitor cells during differentiation. Interestingly we did also detect EGFR amplification in neural stem cells differentiating 
towards the astrocytic lineage. Our array-based amplification analyses warrant a more detailed analysis of gene and chromosome regions to get a deeper insight in gene amplification pattern in stem and progenitor cells.

The amplification data of stem and progenitor cells and the amplification data obtained for glioma stem-like cells, can help to define cell populations at the origin of the glioma stem-like samples. Since MDM4 was only amplified in neural stem cells, neural stem cell are likely to be at the origin of the glioma stem-like cells \#10, \#993 and G112. EGFR amplification in undifferentiated and differentiating NHNP cells, point to neural progenitor cells at the originating of glioma stem-like cell samples. $D D B 1$ amplification was only present in NHNP cells and in neural stem cells that were differentiation induced towards astrocytes. $D D B 1$ copy number increase was present during beginning differentiation of neural stem cells towards neurons (24-48h). During further differentiation of neural stem cells towards oligodendrocytes for 72 $96 \mathrm{~h}$, copy number increased from under-replicated state to normal copy number. These data indicate a more differentiated cell type as originating cell in glioma stemlike cell \#993 derived from oligodendroglioma. GFAP amplification was only detected in neural stem cells and neural progenitor cells but not in differentiation-induced progeny of neural stem and progenitor cells. These data indicate a neural stem and neural progenitor cell at the origin of glioma stem-like samples \#993 and \#1043. In conclusion, gene amplifications specifically gene amplifications of DDB1, EGFR, MDM4 and GFAP can provide information about the cell types and the degree of heterogeneity at the origin of glioma stem-like cells. CDK4, MDM2, GINS2 and TP53 amplifications appeared less or not suitable to narrow down the originating cell composition. It is possible that the tumor reuse the same amplification pattern that is physiologically defined to normal stem cells. While this information can help to learn more about the origin of glioma it will not have immediate impact on new clinical treatment strategies of glioma.

\section{Amplification maintained throughout stem cell divisions}

Interestingly, $C D K 4$ amplification appears to be rather frequent with amplification detected in all investigated samples. Notably, although fluctuations in $C D K 4$ copy number were found during differentiation, the $C D K 4$ amplification was found to be a persistent phenomenon. During differentiation of neural stem cells towards oligodendrocytes, amplification of $C D K 4$ even increased after four days. Fluorescence in situ hybridization of neural progenitor cells indicated $C D K 4$ amplifications in a limited number of neural progenitor cells with asymmetrical cell division (Supplementary Figure 1). The maintained $C D K 4$ amplification in only few cells suggests that $C D K 4$ amplification contributes to the overall regenerative capacity of the whole population. Our results support the idea that basic amplification level of CDK4 is conserved in neural stem cells, in neural progenitor cells, and in their differentiated progeny. It is tempting to hypothesize that $C D K 4$ amplification is important to maintain proliferation and self-renewal propensity not only in neural stem cells but also in their differentiated progenies. Notably, the highest level of $C D K 4$ amplification was found under oligodendrocyteinducing conditions (Table 1). Furthermore, although the increase in CDK4 amplification was detected under all conditions tested at 1 day after differentiation induction, its magnitude was most persistent under oligodendrocyteinducing condition. In this regard, it is noteworthy that $C D K 4$ plays an important role in the maintenance of self-renewal in $\mathrm{NG} 2+$ progenitors that comprise a principal population of proliferation-capable cells for oligodendrocytes in the olfactory bulb [20,21]. Further testing is required to clarify the relationship between CDK4 amplification and self-renewal capacity.

\section{MATERIALS AND METHODS}

\section{Cell culture and differentiation}

NHNP cells were grown and differentiated as described previously [5]. GIBCO Human neural stem cells (H9 hESC-derived) further named NSC, were cultured on CELLStart ${ }^{\mathrm{TM}}$-coated culture ware with complete StemPro NSC SFM medium as described in the manufacturers' instructions. Neural stem cell sphere cultures were grown in uncoated culture ware.

For spontaneously differentiation of NSCs, these cells were plated at $2.5 \times 10^{4}$ cells $/ \mathrm{cm}^{2}$ on CELLStart tissue culture plates in StemPro NSC SFM medium without bFGF and EGF.

For differentiation towards oligodendrocytes NSC were plated at $2.5 \times 10^{4}$ cells $/ \mathrm{cm}^{2}$ on poly-L-ornithine/ laminin coated tissue culture plates with Neurobasal Medium (supplemented with B-27 serum-free supplement, GlutaMAX and 30ng/ml T3).

For differentiation towards astrocytes NSC were plated at $2.5 \times 10^{4}$ cells $/ \mathrm{cm}^{2}$ on Geltrex matrix coated tissue culture plates with D-MEM (supplemented with N-2 supplement, GlutaMAX and 1\%FBS).

For differentiation towards neurons NSC cells were plated at $2.5 \times 10^{4}$ cells $/ \mathrm{cm}^{2}$ on poly-L-ornithine coated tissue culture plates with Neurobasal Medium (supplemented with B-27 serum-free supplement and GlutaMAX).

Glioma stem-like cells (GIC) were isolated from surgical specimens and characterized as described previously $[11,22]$. 


\section{DNA isolation}

Cells were harvested and DNA was isolated using chlorofom $/ \mathrm{NaCl}$ method. In brief cell pellet was digested with Proteinase $\mathrm{K}$ at $55^{\circ} \mathrm{C}$ over night $(>12 \mathrm{~h}$ ) and chloroform extracted for $1 \mathrm{~h}$ at room temperature.

\section{QPCR analysis}

TaqMan Copy Number Assays for genes CDK4 (Hs00957586_cn), MDM2 (Hs00181272_cn), GINS2 (Hs05472641_cn), TP53 (Hs05506931_cn), DDB1 (Hs07226265_cn), EGFR (Hs01463609_cn), MDM4 (Hs05784087_cn), GFAP (Hs01144882_cn) were performed following manufacturers instructions. We used the RNaseP TaqMan Copy Number reference assay for relative quantitation of copy number of target genes. DNA from human normal blood lymphocytes $(\mathrm{PB})$ was used as control standard for normal diploid copy number.

TaqMan assays were run in four technical replicates and results were analyzed using StepOne ${ }^{\mathrm{TM}}$ Software v2.0 and CopyCaller ${ }^{\mathrm{TM}}$ software.

\section{Fluorescence in situ hybridization}

BAC clones were taken either from the RP11 Human Male BAC library, that was generated by the BACPAC Resource Center (BPRC) at the Children's Hospital Oakland Research Institute by Kazutoyo Osoegawa [23] or from RZPD made available through SourceBioSciences, Germany. BAC-DNA $(1 \mu \mathrm{g})$ was either labeled with Alexa488-dCTP, with Alexa-555-dCTP or with Alexa-594-dCTP using the FISHTag DNA labeling Kit according to the manufacturer's instructions. Differentially labeled probe DNAs (60 ng) were precipitated in the presence of human Cot-1 DNA. Samples were resuspended in hybridization mix $(50 \%$ formamide, $2 x \mathrm{XSPE}, 10 \%$ dextrane sulphate and $4 \%$ SDS). Alpha-centromere probe from chromosome 1 (D1Z5) was added to the resuspended probe mix.

Differentiating human neural stem cells and undifferentiated neural stem cells were grown on glass slides with appropriate coating and fixed in ice-cold methanol for 20 minutes. Slides were washed in PBS for 5 minutes and treated with $0.02 \%$ Tween- 20 for 3 minutes. Slides were RNase treated $(100 \mu \mathrm{g} / \mathrm{ml}$ RNaseA in $2 \mathrm{x}$ SSC) for $1 \mathrm{~h}$ at $37^{\circ} \mathrm{C}$ and pepsin treated $(0.005 \%$ in $0.01 \mathrm{M}$ $\mathrm{HCl}$ at $37^{\circ} \mathrm{C}$ ) for 10 minutes. Postfixation was performed using $1 \%$ formaldehyde/1x PBS for 10 minutes at room temperature. Hybridization and posthybridization washes were as described previously [6].

\section{Abbreviations}

array-CGH (array comparative genomic hybridization), BDNF (brain-derived neurotrophic factor), CDK4 (cyclin-dependent kinase 4), CDT1 (Chromatin Licensing And DNA Replication Factor 1), DDB1 (DNAdamage-binding protein 1), EGFR (epidermal growth factor receptor), FISH (fluorescence in situ hybridization), GFAP (Glial Fibrillary Acidic Protein), GIC (glioma stem-like cell), GINS2 (go-ichi-ni-san 2), hESC (human embryonic stem cell), MDM2 (mouse double minute 2), MDM4 (mouse double minute 4), NG2+ (nerve/ glial antigen 2 positive), NHNP (normal human neural progenitor), NSC (neural stem cell), PB (peripheral blood), qPCR (quantitative polymerase chain reaction), SFM (serum free medium).

\section{Author contributions}

UF performed experiments, designed the study and wrote the manuscript. EK performed experiments with glioma sphere cells and supplied DNA. AK analyzed data and reviewed the manuscript. EM designed the study and wrote the manuscript.

\section{ACKNOWLEDGMENTS}

We thank E. Maldener for technical assistance.

\section{CONFLICTS OF INTEREST}

The authors declare no conflict of interests.

\section{FUNDING}

The "Deutsche Forschungsgemeinschaft" funded this study (Fi644/2-1; Fi644/2-2).

\section{REFERENCES}

1. Schwab M. Oncogene amplification in solid tumors. Semin Cancer Biol. 1999; 9:319-25.

2. Fischer U, Leidinger P, Keller A, Folarin A, Ketter R, Graf N, Lenhof HP, Meese E. Amplicons on chromosome 12q13-21 in glioblastoma recurrences. Int J Cancer. 2010; 126:2594-2602.

3. Meinhardt G, Kaltenberger S, Fiala C, Knöfler M, Pollheimer J. ERBB2 gene amplification increases during the transition of proximal EGFR(+) to distal HLA-G(+) first trimester cell column trophoblasts. Placenta. 2015; 36:80308 .

4. Hannibal RL, Baker JC. Selective Amplification of the Genome Surrounding Key Placental Genes in Trophoblast Giant Cells. Curr Biol. 2016; 26:230-36. 
5. Fischer U, Keller A, Voss M, Backes C, Welter C, Meese E. Genome-wide gene amplification during differentiation of neural progenitor cells in vitro. PLoS One. 2012; 7:e37422.

6. Fischer U, Backes C, Raslan A, Keller A, Meier C, Meese E. Gene amplification during differentiation of mammalian neural stem cells in vitro and in vivo. Oncotarget. 2015; 6:7023-39. doi: 10.18632/oncotarget.3248.

7. Fischer U, Ludwig N, Raslan A, Meier C, Meese E. Gene amplification during myogenic differentiation. Oncotarget. 2016; 7:6864-77. doi: 10.18632/oncotarget.6845.

8. Fischer U, Ludwig N, Keller A, Backes C, Meese E. Genome-wide copy number profiling of mouse neural stem cells during differentiation. Genom Data. 2015; 5:3-6.

9. Anker L, Ohgaki H, Ludeke BI, Herrmann HD, Kleihues P, Westphal M. p53 protein accumulation and gene mutations in human glioma cell lines. Int J Cancer. 1993; 55:982-987.

10. Kim E, Günther W, Yoshizato K, Meissner H, Zapf S, Nüsing RM, Yamamoto H, Van Meir EG, Deppert W, Giese A. Tumor suppressor p53 inhibits transcriptional activation of invasion gene thromboxane synthase mediated by the proto-oncogenic factor ets-1. Oncogene. 2003; 22:7716-27.

11. Barrantes-Freer A, Renovanz M, Eich M, Braukmann A, Sprang B, Spirin P, Pardo LA, Giese A, Kim EL. CD133 Expression Is Not Synonymous to Immunoreactivity for AC133 and Fluctuates throughout the Cell Cycle in Glioma Stem-Like Cells. PLoS One. 2015; 10:e0130519.

12. Sher N, Bell GW, Li S, Nordman J, Eng T, Eaton ML, Macalpine DM, Orr-Weaver TL. Developmental control of gene copy number by repression of replication initiation and fork progression. Genome Res. 2012; 22:64-75.

13. Douglas ME, Diffley JF. Recruitment of Mcm10 to Sites of Replication Initiation Requires Direct Binding to the Minichromosome Maintenance (MCM) Complex. J Biol Chem. 2016; 291:5879-88.

14. Jin J, Arias EE, Chen J, Harper JW, Walter JC. A family of diverse Cul4-Ddb1-interacting proteins includes Cdt2, which is required for $\mathrm{S}$ phase destruction of the replication factor Cdt1. Mol Cell. 2006; 23:709-21.
15. Meletis K, Wirta V, Hede SM, Nistér M, Lundeberg J, Frisén J. p53 suppresses the self-renewal of adult neural stem cells. Development. 2006; 133:363-69.

16. Middeldorp J, Boer K, Sluijs JA, De Filippis L, EnchaRazavi F, Vescovi AL, Swaab DF, Aronica E, Hol EM. GFAPdelta in radial glia and subventricular zone progenitors in the developing human cortex. Development. 2010; 137:313-21.

17. Mohamad Ashari ZS, Sulong S, Hassan R, Husin A, Sim GA, Abdul Wahid SF. Low level of TERC gene amplification between chronic myeloid leukaemia patients resistant and respond to imatinib mesylate treatment. Asian Pac J Cancer Prev. 2014; 15:1863-69.

18. Mansuet-Lupo A, Zouiti F, Alifano M, Tallet A, Charpentier MC, Ducruit V, Devez F, Lemaitre F, Laurent-Puig P, Damotte D, Blons H. Intratumoral distribution of EGFR mutations and copy number in metastatic lung cancer, what impact on the initial molecular diagnosis? J Transl Med. 2014; $12: 131$.

19. Tattini L, D'Aurizio R, Magi A. Detection of Genomic Structural Variants from Next-Generation Sequencing Data. Front Bioeng Biotechnol. 2015; 3:92.

20. Jablonska B, Aguirre A, Vandenbosch R, Belachew $\mathrm{S}$, Berthet C, Kaldis P, Gallo V. Cdk2 is critical for proliferation and self-renewal of neural progenitor cells in the adult subventricular zone. J Cell Biol. 2007; 179:123145.

21. Aguirre A, Gallo V. Postnatal neurogenesis and gliogenesis in the olfactory bulb from NG2-expressing progenitors of the subventricular zone. J Neurosci. 2004; 24:10530-41.

22. Barrantes-Freer A, Kim E, Bielanska J, Giese A, Mortensen LS, Schulz-Schaeffer WJ, Stadelmann C, Brück W, Pardo LA. Human glioma-initiating cells show a distinct immature phenotype resembling but not identical to NG2 glia. J Neuropathol Exp Neurol. 2013; 72:307-24.

23. Osoegawa K, Woon PY, Zhao B, Frengen E, Tateno M, Catanese JJ, de Jong PJ. An improved approach for construction of bacterial artificial chromosome libraries. Genomics. 1998; 52:1-8. 